\title{
DOI: https://doi.org/10.24297/jap.v20i.9179
}

\section{The second revolution of relativity}

\author{
A.Merouani \\ Ex-researcher, in Ferhat Abbas University-Sétif-ALGERIA \\ Ex-Professor, in El Bachir el Ibrahimi University-Borj Bouareridj-ALGERIA \\ /amerouani2001@yahoo.fr
}

\begin{abstract}
Energy, this vital entity that we need, and that everyone consumes and liberates, in different forms, has been since the dawn of time the greatest concern of the human being. Over the centuries, energy has transformed lifestyles in all societies of the world, contributing to the emergence of new cities and major cosmopolitan cities. Scientific research has contributed to much of this extraordinary advance of energy in the conquest of the world, thanks to the many works and discoveries of scientists from the golden age of Islamic civilization to the renaissance in Europe, to the great technological revolution that has emerged in the North and in the South. The continuous need for energy has always been the main driver, for various discoveries, whether related to energy matter or those related to energy reactions. Between pure matter and an active reaction, energy has become the focus of several hybrid studies, manipulating energy as a source on the one hand, and as a finished product on the other.

While most of the work carried out on the electric current, during the first years of the 17th century [1], showed the precariousness of the materials as regards their conductivity, the arrival on the scientific scene of new chemical elements, discovered then around the 18th century, have strengthened the quality of the works and consequently the improvement of the electrical productivity in the world. Nuclear energy will suffer the same fate of development as that of electrical energy, passing through critical periods, especially those relating to the discovery of high-activity materials [2]. Nevertheless, the arrival at the beginning of the 18th of the famous formula of Albert Einstein on relativity, $\left(E=m c^{2}\right)$ [3], put an end to the major concerns of scientists on energy, to know finally, the nature and value of the energy compensation of the material during its activity.

We, in turn, consider that Albert Einstein's formula is not entirely sufficient to decipher the true energetic reality of matter. Our work is a re-shaping of the relativistic formula, not only from the physical point of view but also from the mathematical point of view. As a result, our contribution to this restoration pleads for a new apprehension of the atom as an «original copy of the universe» which has an infinity of nanoscopic particles, which remain active within matter, Chemically active and physically metastable. This extrapolation of the relativistic formula will recognize to energy the other side of its independence from matter.
\end{abstract}

Keywords: Relativity, Thermodynamics, nanotechnology, energy, the black nano holes.

\section{I-Introduction}

In the mid-19th century, the emergence of the concept of energy conservation gave rise to one of the great controversies of classical physics, opposing mechanism and energy. Since then, several researchers have been working on advancing energy conservation theories and laws. They include James Prescott Joule, Christian Huygens and William Hamilton [4]. Each developed the real mechanisms of heat transfer, mechanical and thermal energy. The criteria thus developed to define and defend forms of energy conservation were based solely on mechanical labour force, which governs all forms of atomic motion and subsequently generates quantum energy. The various forms of energy conservation are, in our opinion, insufficient to control the development of research at the level of the science of nanometric particles, because of the failure to take charge in energy conservation formulas, inter-particle friction movements that generate quantum dissipative nano-systems, which exchange energy and matter, thus embodying a nanometric thermodynamic model.

The world of nanotechnology opens up several research options on the value of energy and its real and unlimited sources. Einstein's relativistic formula had as its purpose the demonstration of the existence of energy from matter, where as nanotechnology should, according to the fundamental laws of physics, prove the existence of energy from the immaterial. The notion of heat and light, are a concrete example of this immaterializations of the energy source. Considering that the phenomena of heat diffusion in a reaction medium are subordinated to the existence of mass differences and that light diffusion in an inert medium is subordinated to the difference in potential, We conclude 
that there is no compatibility between heat and light, even though they are considered as energies!. Moreover, we cannot judge on the nature of energy only if and only if we know the fundamental properties of matter. These fundamental properties, however, are unrecognizable at the nanoscale.

According to Einstein's formula $E=m c^{2}$, there is a balance between energy and matter which is justified by the law of mass conservation. However, if this formula is extrapolated to the nanoscale, this will not be the case, because the balance would be due not to the law of mass conservation; but to the law of mass energy conservation. This implies that at the nanoscale the systems of heat flux exchanges are open, and therefore it is a boon for researchers to revolutionize quantum laws, involving new thermodynamic parameters. A new era of "nanoscale thermodynamics" will then emerge.

\section{II-Nanometric thermodynamics}

Starting from the first principle of thermodynamic, we confirm that all energies have the same physical transfer property, which allows them to energize the systems involved. Apart from this physical quality, energies diverge in their states. Modern physics is reproached for taking energy as a consequence of the safe guarding of mass, when in reality energy is a cause of the safeguarding of mass. That is to say, the conversion of mass into energy does not depend on our will, not on that of matter, and that this conversion action is «quantum instructed» and that it cannot create anything other than energy. The question we ask, is whether energy could manifest in other forms than those we know of? ,. The ability to know its new forms of energy depends on our conception of the universe and its components. Through its distinction on other disciplines related to the science of matter, nanotechnology, allows thanks to the less dense surfaces of nanoparticles to amplify the absorbance of light spectra ( from radioscopic generators), and therefore encourage the development of new, thermodynamically active plasmas.

By characterizing the disorder within plasmas as a metastable state, such as entropy within classical thermodynamic systems, this new group of plasmas is considered to be separate systems that move in a different environment than the parent nanoparticles. The modification of atomic tissues within nanoparticles, by radioscopic effects, is a clear answer to the enigmatic questions about the reality of matter. This physical phenomenon is purely similar to that of nuclear fission, which generates energetically active particles. As soon as we discovered that matter could decompose ; not only to give new particles similar to the source, but that it could generate energy-rich systems that coexist in matter itself. It is clear that energy conservation theory is becoming more important in nanoscale thermodynamics.

The nanometric universe is particularly different from the microscopic universe in that it is oscillations, vibrations and deformations of atoms. The different electronic structures of the chemical elements prove that there is a considerable link between the nuclei and their environment. Electronic distribution, in our opinion, would not be included by chance in the chemical elements, but it would be due, during the creation of the universe, to the taking of the electronic cloud of atoms between two movements, one related to magnetic fields emanating from nuclei, and the other related to an unquantified energy environment. The balance thus created within the electronic balance directly influences the thermodynamic balance of the systems. Therefore, any disorder within the electronic clouds would create a disorder in the systems that would result in the questioning of all forms of energy.

\section{-III-Nano black holes}

As already mentioned above, we consider that the atom is a copy of the universe. The electronic equilibrium within the chemical elements is due to the opposite motions of magnetic fields from the nuclei and the unquantified environment. The latter is considered to us, as the black nano-holes that correspond to those that exist within galaxies [5] . Maximizing nanometric research would make it possible to identify these nano-holes, which are unknown today by physicists. The nano holes would therefore be, the unquantifiable environment that represents the force opposite to that of the magnetic force of the nucleus. It is an imperceptible energy environment, due to its high frequency of emission. It is an imperceptible energy environment, due to its high frequency of emission. Physically speaking, black holes are part of the atom, but chemically they are not.

\section{IV- Quantum description and role of black nano holes}

If nuclear power has been able to prove the energetic strength of the nuclei, it has unfortunately not found solutions to the manipulation of the atom in the direction, to make it energetically ineffective in order to minimize the devastating effects of its manipulation. ! The reasons for this failure is due to the very important delay of the research in the discovery of the true secrets of the atom. This is not because researchers do not want to find technical or peaceful solutions to nuclear weapons, but because they lack basic information about the atom in order to verify the possibility of an imminent 
discovery relating to the destruction of nuclear energy within the atom itself before any manipulation, or even after the manipulation of atomic nuclei. If the name of the nuclei is "white matter," then the name of "dark matter" is attributed to the black nano-holes. Between these two materials exists the electronic cloud. If the chemical effects of heavy nuclei are assigned to white matter, we consider that the physical effects are assigned to dark matter. This could well be verified in the case of nuclear reactions, where the set of reaction products are new atoms associated with energetic particles; without, however, giving the reasons for the passage of atoms to a second stable state, despite there has been the degeneration of white matter!._One could explain this shift to a new stability, to the condensation of electronic clouds, because of the inductive effect of dark matter, and consequently their physical inertia, which explains the rather long life of heavy metals, from atomic decay. Without a doubt, we can say that the speed of deformation of the electronic cloud is faster than the speed of disintegration of nuclei, which accelerates the formation of new nuclei, but if it were the opposite, there will be no more new nuclei.

\section{V- The nano hole model}

We have so far insisted in our article, on the existence of dark matter within the atom. The model we design for nano holes within the atom is as follows:

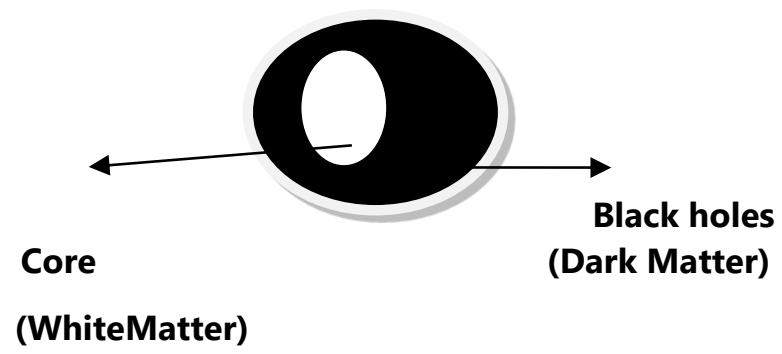

Fig.01 Theoretical model of the atom

updating the existence of these black holes would promote the appearance of a new form of material physics, involving the interference of two materials within the atom, This would lead to the involvement of new variables in the general formula of mass preservation. A correct and real correction of the physical calculation, long under suspicion of their accuracy, would then be possible and acceptable. In quantum physics, the density matrix, is a mathematical object introduced by the mathematician and physicist John von Neumann to describe the state of a physical system [6]. If we gave another form to the atom, then the physical system would have to meet the new conditions of existence of the atom below. Thus, if the scientist von Neumann described the density matrix as the summation of all the states of the system [7], which are in connection with the nucleus (white matter), it would be more descriptive in our case to add to this summation the state of the physical system that defines dark matter. We therefore consider that in the atom there are two physical systems and not one. Mathematical calculation often provides information on the forms and number of physical states of systems, but does not provide information on the inequality of energy ratios within the same atom. The opaque nature of the energy transfer regimes within the nuclei, currently hinders the real explanation of the disappearance of matter without quantifying the exact value of the equivalent energy. The simple manipulation of the atom for the purpose of appropriation of its energy capital, does not exclude the partial mutation of the energy released into another electromagnetic waveform!.

\section{VI - Energy surplus}

If the universe as a whole is still moving towards infinity in space and towards the limit in time, then the mathematical projection of its movement would be a fairly simple equation, where the sum of energies Liberated (El) at the second by the integrated particles of this universe is equal to: the total energy consumed $(E c)$ and the total energy stored (Es). Or as follows:

$E I=E C+E s$ ....... Eq-01

Two relevant questions arise:

- Question 1: Who consumes this released energy?

- Question 2: Where is the energy not consumed stored and why was it not consumed?

This principle takes us back to the atom that was said to be a copy of the universe and goes back to the two previous 
questions:

- Question 1: Who consumes the energy released by the atomic nucleus?

- Question 2: Where is the energy not consumed stored and why was it not consumed?

By a methodological analysis, we can prove that the universe has an energy surplus, in the same way for the atom, which also has an energy surplus.

How?

As a matter of principle, we all agree that any chemical, electrochemical or nuclear reaction should emit desired products and others called by-products, in liquid or gaseous solid form, which appear because of a bad reaction environment or because of unwanted side reactions. These by-products are eliminated either by physico-chemical methods or by spectroscopic methods. But if this by-product is energy, it cannot be disposed of, whether it is stored within the atom or released and not consumed!. We should accept that all energy consumption has limits, whether it is for reasons of life, or for other reasons of death! In this vision, we should therefore divide the concept of energy into three categories, positive energy, negative energy and stored energy. All its three energies form, in our opinion, a battery of life that governs the universe.

\section{VII- The new physical model}

The three energy categories are defined by the following abbreviations:

Negative energy, which is released by: $\mathrm{E}-$.

Positive energy, that which is consumed by: $E$ +. Stored energy, rated stored energy by: E S

Quantum speaking, the energetic movements are as follows:

M (Pure material) $\quad$ E - + M+

\section{(Degraded material)}

$\mathrm{U}$ (Universe) $+\mathrm{E}+\quad \longrightarrow \quad \mathrm{U}-$

Global Response:

$\mathrm{M}+\mathrm{U}+\mathrm{E}+\longrightarrow \mathrm{E}-+\mathrm{M}++\mathrm{U}-$

$\mathrm{Eq02.}$

According to equation 02, we notice that the universe/Matter couple is in an infinite race towards the degradation of matter and the transformation of the universe. This exponential depletion of pure matter, and the logarithmic enrichment of the universe, with a variation of stored energy, brings us back to propose the following mathematical model, relating to a new equation of energy conservation:

If $(t)$ is the time we will have:

e $\mathbf{M}^{+} \mathbf{t}+\log \mathbf{U}^{-} \mathbf{x t}-\mathbf{E s}=\mathbf{0}$ Eq03.

This equation translates, a physical model not applied today, and which involves in addition to living matter, a variable and constancy, in this case the universe and the stored energy.

From equation 03 , we determine the equation that defines : $U_{-}$

By posing $\mathrm{m}+=\mathrm{X}$, and $\mathrm{U}-=\mathrm{Y}$, we will have; equation 3 in the form:

$e X t+\log y-E s=0$ Equ04.

Hence $y=e^{\text {Es-ext }} \ldots .$. Eq05.

Boundary calculations show that:

$A t=0 \quad y=e E s$ 
At $=\infty \quad y=e E s$

This borderline study clearly shows that the universe is a constant entity over time, and that it would never evaluate, despite the thousands of chemical or nuclear reactions that could occur, given its immensity and greatness! . Another more important and practical result is that of stored energy, which would also be more important of the energy of the universe, and more important than infinity!., which suggests that black holes would be the largest sources of stored energy.

This correlation between the universe and the stored energy proves the existence of a sensitive and critical energy balance between these two elements.

By replacing log y as a constant, and which represents the energy of the universe, which we designate as Eu, in equation 04, we will then have:

$e^{X t}+E u-E s=0$ Equ06.

Hence: eXt = Es-Eu this implies:

$X t=\log (E s-E u)$ this implies

$X=\log (E s-E u) / t$

$X=\log \Delta E / t$ Eq07

By measuring unites, we find that $\mathrm{X}$ represents a power of degraded dark matter, which we represent by $\mathrm{P}$.

So we'll have:

$P_{h}=\log (E s-E u) / t$ Eq08.

$P_{h}=\log \Delta E / t$ .Eq09

\section{Diagram of the model}

The schematic of the new model is identical to the one assigned to the atom. So the nucleus represents the earth and the dark part the universe. Nevertheless what is remarkable?

\section{Dark Matter}

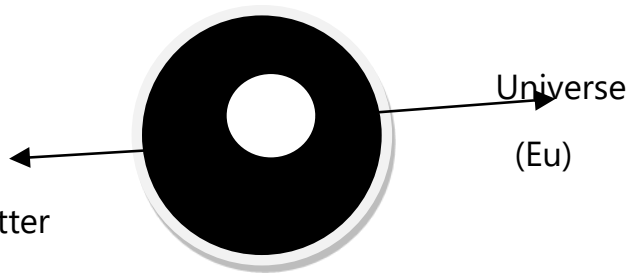

(Es)

If at the atomic nucleus level we have, the particle power is given by the expression:

$-\mathrm{Pn}=\Delta \mathrm{E} / \mathrm{t}$ as $\mathrm{Pn}$ is the particle power

And at the level of the universe in general: as $\mathrm{Ph}$ is the power of black holes 
Hence we have:

$-\Delta E_{n}=$ Pnx $t$ Eq10.

$-\Delta E_{h}=t \times e^{P h}$. Eq11

\section{Conclusion}

According to equations 10 and 11, it is noted that If Einstein's relativity made it possible to determine the secondary state of matter after its disintegration, the second relativity that we propose demonstrates the state of matter of black holes during their evolution, So if for Einstein, energy evolves in proportion to matter, for us the energy of black holes evolves exponentially with matter. In conclusion, the black holes would be gigantic deposits of energy that would far exceed that of the universe and the earth.

\section{REFERENCES}

1. Heilbron, John L., Electricity in the 17th and 18th Centuries: A Study in Early Modern Physics, New York: Dover, 1999, p. 169-179.

2. S.Rosenblum, « l'aspect historique de la découverte de la radioactivité artificielle ».Journal de physique et de radium ; tome 16 1955. https://doi.org/10.1051/jphysrad:019550016010074301

3. J. L. Synge, Relativity, the general theory. North Holland Pub., 1960

4. Patricia Radelet De Grave ; Histographie de la mecanique de 1975-200 : Etude Statistique. ILUIL ; Vol. 26, 2003, 995-1044

5. Almheiri Ahmed, Donald Marolf, Joseph Polchinski et James Sully, «Black holes: complementarity or firewalls? », Journal of High Energy Physics, février 2013 . https://doi.org/10.1007/JHEP02(2013)062

6. Salim Rashid; John von Neumann and Scientific Method. Journal of the History of Ideas. Vol. 68, pp. 501-527; No. 3, Jul., 2007. https://doi.org/10.1353/jhi.2007.0025

7. Miklós Rédei and Michael Stöltzner; John von Neumann and the Foundations of Quantum Physics. Vienna Circle Institute Yearbook [2000] book serie (VCIY, volume https://doi.org/10.1007/978-94-017-2012-0 